International Journal of Engineering \& Technology, $7(2.7)(2018) 175-178$
International Journal of Engineering \& Technology
Website: $w$ ww.sciencepubco.com/index.php/IJET
Research Paper

\title{
IoT based reconfigurable smart city architecture
}

\author{
M. Geetha Pratyusha ${ }^{1 *}$, Yogesh Misra², M. Anil Kumar ${ }^{3}$ \\ ${ }_{1,2}$ Department of Electronics and Computer Engineering, \\ ${ }^{3}$ Department of Electronics and Communication Engineering, \\ Koneru Lakshmaiah Education Foundation, Vaddeswaram, Guntur, Andhra Pradesh, India - 522502 \\ * Email: geethapratyusha454@gmail.com
}

\begin{abstract}
Now-a-days Internet of Things is deployed almost in every application regarding smart cities and have been initiated to develop all over the world. Smart Devices are being geared up to ease human life. Due to the growth in Internet of Things technology, smart cities are been developed with this technology to work with the issues of public as well as private. The aim of the article is to enhance a solution to the problems in the smart cities with latest IoT architecture, protocols and services. With the technical support of IoT, using low power Wireless Sensor Networks (WSN) which is connected to transfer the data from M2M applications. In addition to the IoT, intelligent features are integrated with the help of Computer vision makes the technology more flexible. The goal of this article is to engrave the services to challenge the real time environment with low power synthesis technique. Only then, the smart city features are improved and serves the mankind with IoT technology.
\end{abstract}

Keywords: Internet of Things, Computer vision, Wireless Sensor Networks, M2M applications, low power synthesis technique.

\section{Introduction}

\section{A. Concept Orientation}

IoT Technology has been improved with the services and infrastructures to provide the basic requirements in the urban cities. With the observation of Gartner,50 billion objects will be connected and incorporated with the smart cities by 2020[1]. With the enhancing IoT technology we are integrating the device with this technology like smart watches, smart TV and Internet boxes. To drive huge business potentials, all these devices are communicated internally using IoT Technology and these devices are most self-configuring and smart objects with global network Infrastructure [2].

\section{B. Motivations}

A Smart city is highly incorporated with different digital devices using communication and networking protocols [3]. These devices are controlled to make the governance-oriented applications like smart street, smart traffic etc. In addition, these devices are improved with Computer vision to provide more features to the devices in the heterogeneous environment. Sensors in the devices collect data from several applications from vehicles, human health, parking etc. These devices make revolution by interconnecting with various objects using Internet. The main outlook of IoT Based Reconfigurable Smart City Architecture is shown in Fig. 1.

IoT can also play a pivotal role regarding to the policy decisions and remote monitoring the services [4]. On these bases, IoT will be deployed almost in different aspects to make the devices more stable, effective and economic to the world.

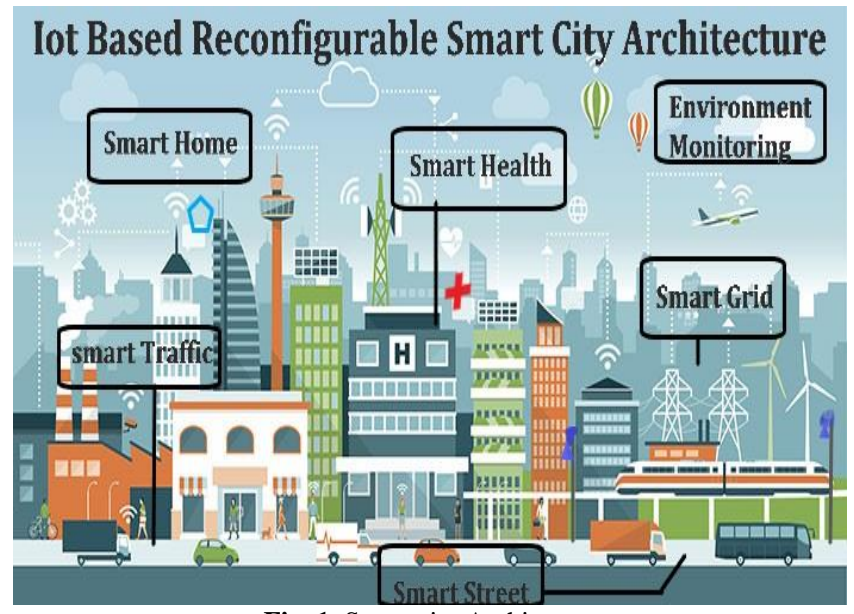

Fig. 1. Smart city Architecture

\section{IoT Architecture}

To provide an IoT for a device, complexity to simplicity is more required to make the one to many heterogeneous devices, with sensors that produce data. This makes the analyzation to gain insights [5]. IoT devices are connected directly to a network or a gateway which enables the communication with each other and it is stored at cloud services to make more accessibility from device to device are shown in Fig. 2. 


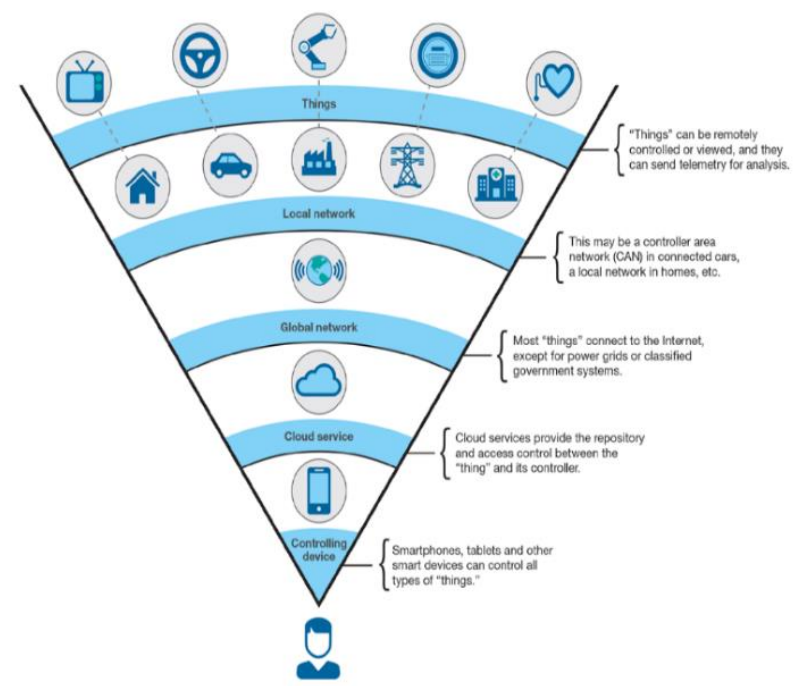

Fig. 2: Device to Device Communication along with Cloud Service

IoT Architecture plans following strategies to provide simplifying developments, manage strategies and ensure that IoT solution is robust and flexible. Most architectures are included with physical features such as sensors, devices and virtual features such as communication protocols adopts multi-tiered layered architecture [6] are shown in the Fig. 3.

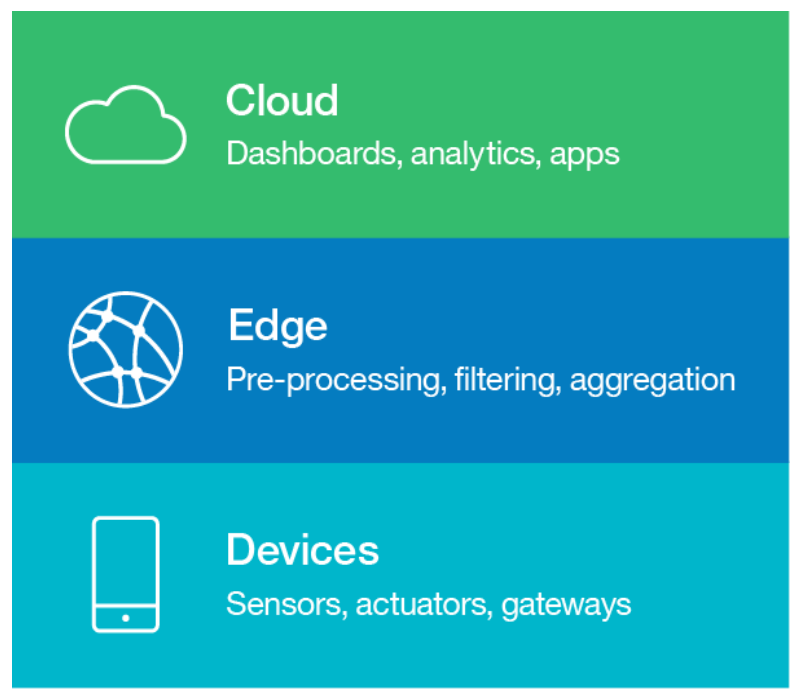

Fig. 3. Multi-Tiered Layered Architecture

\subsection{Device Layer}

Sensors/Actuators are used to sense the data in their own physical way and connect either directly or wireless over the technologies like BLE, ZigBee, and Wi-Fi. These data from sensors could be analog form also which can be gained using analog to digital conversion or digital form. These devices directly communicate with the cloud using Gateway orientation. Gateway plays an important role in data acquisition.

\subsection{Edge Layer}

After data acquisition from the device layer, edge analysis occurs in real time to process the data from layer point to Edge point. Basic preprocessing tasks like Up streaming /Down streaming are performed to send data to the cloud and receive the data from the cloud.

\subsection{Cloud Layer}

After preprocessing the data to the cloud applications, the data can be accessed to the end users complemented with mobile apps or web application. We can also secure and access data for further exploitation through dashboard and visualization. These are different clouds used in the smart cities to provide more reliability. There are different cloud platforms mostly used according to 2016 are shown in Fig. 4

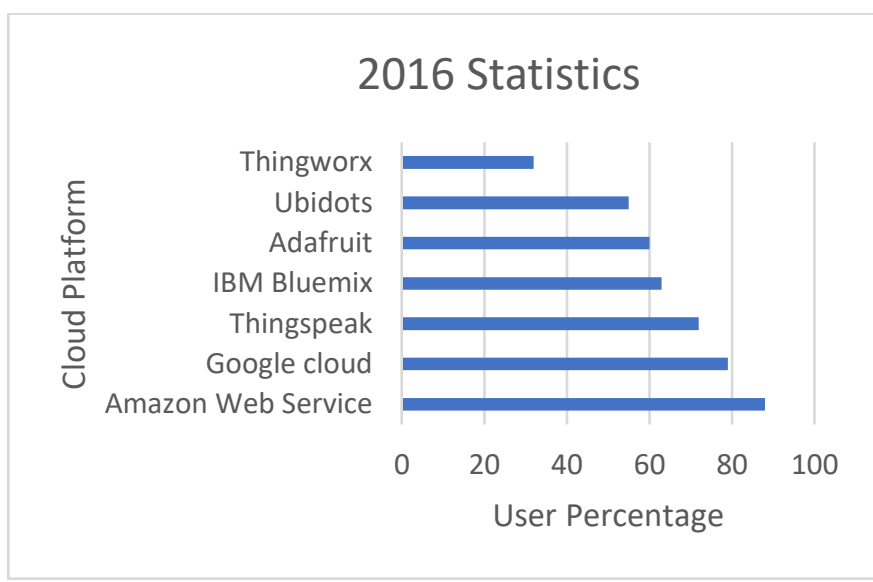

Fig. 4. Statistics of most used Cloud Platform

\section{IoT Based Smart City Applications}

The target to be achieved, is to collect and analyze the data from sensors used for different applications and process to the cloud using low power synthesis technique. Through this technique the wireless interface between device and cloud will consume low power and makes the smart city applications more compatible to the real time environment. Fig. 5. illustrates the area of knowledge for the main smart city applications to be developed.

\section{Smart Home}

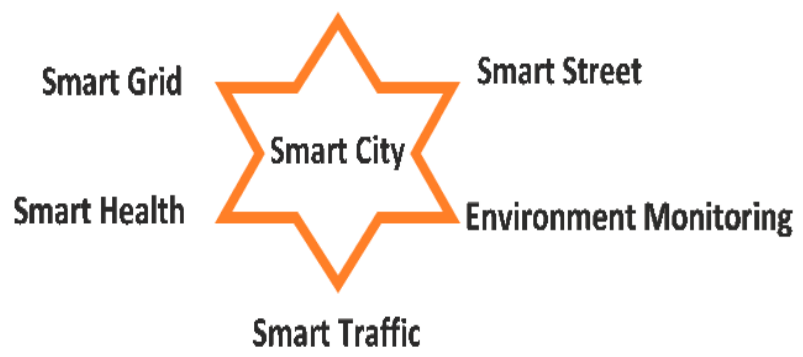

Fig .5. Smart City applications

The proposed system is to develop Smart applications useful for the cities with low power wireless technologies. The application meant for exchanging the data are used with Zigbee (IEEE 802.15.4 protocol) and data upstream/downstream to the cloud is used with low power Wi-Fi module (IEEE 802.11 protocol) affects to create a strong performance.

\section{A. Smart Home}

Smart homes are monitored and controlled using different sensors. Most innovated concept is to develop the smart homes using Google assistant or Alexa and enhance the Computer Vision to control the entry of the house [7]. This improves the Demand Response(DR) of the citizens to incorporate with IoT Technology in the future.

\section{B. Smart Street}

In most of the cities, the public almost face problems due to parking and more energy is consumed due to street lighting. In Smart Parking System, Zones are maintained to provide the specific allotment 
for each vehicle. By making the zone, the vehicle owner gets the information to opt the parking lot. This information will save the traffic to get struck at parking point. For Smart Lightning system, most cities are consuming the electricity at street lighting points. According to a survey $19 \%$ electricity is consumed for lighting [9] With Automatic light system using sensors, governance can save up to $12 \%$ of energy [8].

\section{Environment Monitoring}

Multiple environmental conditions are covered using Wireless Sensor Networks [10].

The parameters to provide information by sensors are:

- Gas concentration in the cities.

- Temperature, pressure and humidity report in the city.

- Heat Detection in the forest areas.

- Forecasting rain report for weather monitoring.

\section{Smart Traffic}

Traffic is one of the most considerable issues, but solving this issue makes beneficial to vehicle users. Traffic occurs due to several reasons like pits, construction barriers etc. In this application, citizens and government gets the opportunity to raise a complaint to resolve the traffic restrain. If the public over - rule the traffic, OBR algorithm using computer vision is used to get the license number and processed with penalty. Smart Vehicle Black box is used in the vehicles to decrease the accident rate in the traffic.

\section{E. Smart Health}

Low cost WSN technologies is applied to monitor the patient health in residential, work and hospital environment. The miniature sensors are incorporated on the human body to know the physical parameters like body temperature, blood pressure [11]. These collected data is analyzed and sent to cloud with low Wi-Fi technology. The sudden change is observed by the server and immediate response is provided to the patient using IoT Technology.

\section{F. Smart Grid}

Smart grid manages two-way communication between a user and power utilizes, which can able to create a distributed and automation energy delivery network. The demonstration can be done in smart home application to bring the cost-effective power generating smart grid system.

\section{Power-Aware Architectural Synthesis}

Low power system synthesis mainly derived from the hardware and software co-synthesis. IoT related applications mostly consider the performance evolution [12]. Wireless Network is mainly focuses on parameter like computational price, speed and power of the System. IoT Based Reconfigurable Smart City Architecture is applied with the "Generic Algorithm Technique" to deal with real time applications. System synthesis optimization flow [13] is shown in the Fig. 6.

In Generic algorithm called COWLS targets embedded systems consisting low power wireless devices communicate with each other e.g., Zigbee, BLE etc. With the usage of data transmission, low energy wireless devices are used from server to client. The technique provides a numerous solution while using the architectural features such as price, power etc. Using this technique, devices transmit data via wireless network protocols with energy minimization. This provides fast and efficiency results to the smart application using the system synthesis.

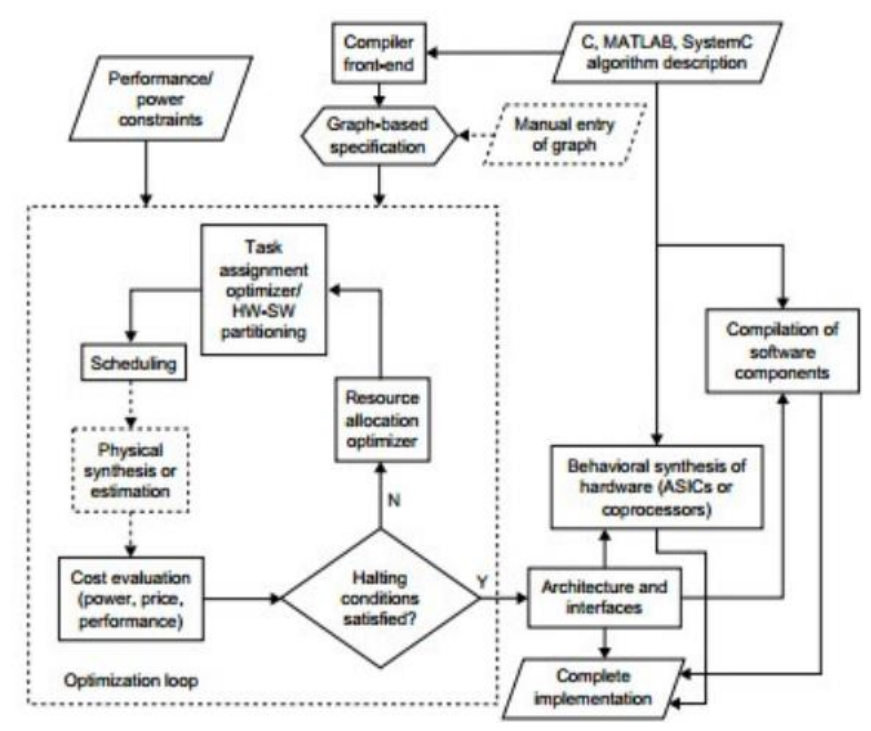

Fig. 6. System synthesis optimization

Low power system synthesis technique for different wireless networks is shown in Table. 1 .

Table I: Low Power Synthesis for Wireless N/W

Table I: Low Power Synthesis for Wireless N/W
\begin{tabular}{|l|l|l|l|l|}
\hline $\begin{array}{l}\text { Protocol } \\
\text { IEEE }\end{array}$ & Wireless Network & $\begin{array}{l}\text { Sleep } \\
\text { mode } \\
(\mathrm{uW})\end{array}$ & $\begin{array}{l}\text { Tx } \\
\text { Power } \\
(\mathrm{mW})\end{array}$ & $\begin{array}{l}\text { Rx } \\
\text { Power } \\
(\mathrm{mW})\end{array}$ \\
\hline 802.15 .1 & Bluetooth (HC-05) & 09 & 39 & 37 \\
\hline 802.15 .4 & Zigbee & 12 & 52 & 54 \\
\hline $802.11 \mathrm{~b} / \mathrm{g} / \mathrm{n}$ & Wi-Fi(ESP 32) & 05 & 190 & 95 \\
\hline
\end{tabular}

\section{IoT Challenges}

IoT Technology main goal is to fulfill the priorities for the smart applications with reliability, security, scalability and performance.

\section{i. $\quad$ Reliability:}

IoT Technology is most improving resource development to any application in the World [12]. Reliability is subject to provide service successfully at any circumstances. This reliability will be more critical in the field of emergency response application.

\section{ii. $\quad$ Security and Privacy:}

IoT system in heterogeneous networks, loss the lack of providing security and privacy. According to the Xu et al. [12] The important features regarding security are authentication, data tracking, Information integrity are not updated in IOT. Billions of objects are distributing information should be encrypted. According to this era, IOT needs to upgrade Security in this field is must necessary for the future.

\section{iii. $\quad$ Big data Analytics:}

50 Billion Device certainly estimated to be interconnected by 2020 . Hence huge storage is necessary to transfer and recall the data. Data should be analyzed well to the further usage [4].

\section{iv. Scalability:}

The system can be extended with new modules, services and technologies for the applicants for better quality of the product Scalability should be handled according to the requirement of the production. Therefore, Customers approach increases with the ease utilization of hardware and software configuration in IoT technology. 


\section{v. WSN Design:}

Smart Sensors are opted to embed with IOT applications. In future the devices should be easy to perform with power to sense, compute and communicate [13]. Sensor nodes need low power to consume but still wireless protocols like BLE, ZIGBEE and Wi-Fi connection require battery either replacement or charging. Despite software challenges, hardware needs to achieve the solution of long term usage [14].

With the advance power aware techniques, devices are produced with low power and cost, makes an IoT product for smart home, smart health etc. more economic. Another constrain is to provide long range communication for the devices [15].

\section{Results and Discussions}

Based on U.S, IoT is an emerging technology and keep on growing in heights until 2025. As mentioned, number of interconnecting devices almost surpassed the population in 2011. Achievements and efforts are established between broad bands are made by the cities Calgary (Canada), Seoul (South Korean), Glasgow (Scotland), Georgia(USA), Singapore and Japan. These are practical results of Smart IoT Applications are shown Fig. 7.

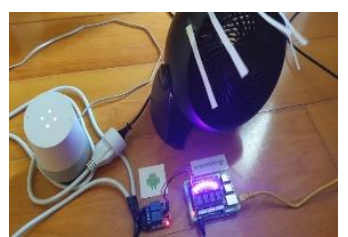

Fig. 7(a).Smart Home

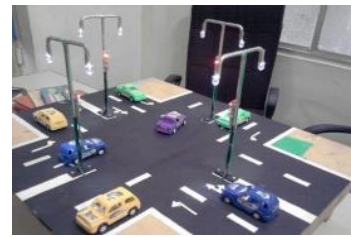

Fig. 7(b).Smart Street

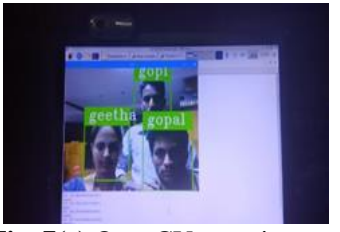

Fig. 7(c).OpenCV-security

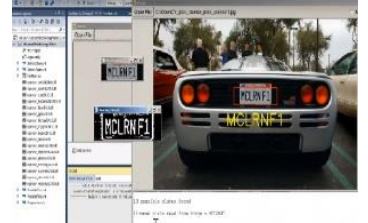

Fig. 7(d).Smart Traffic
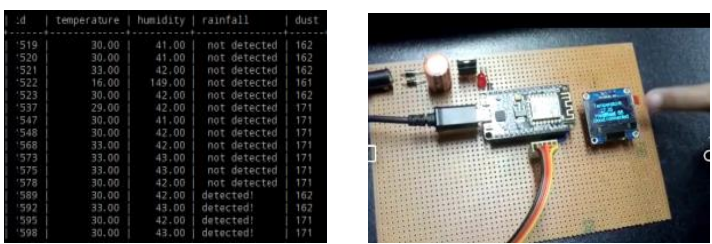

Fig. 7(e).Environment monitoring

Fig. 7(f).Smart Health

\section{Conclusion and Future Trends}

With the increase in the growth of the population, the smart ideas regarding cities becomes vital. The characteristics and features of IoT Technology develop and explain with multiple scenarios like Environmental monitoring i.e., Gas level concentration, Humidity, Temperature and Rain Forecasting, Smart Lighting and Smart parking etc. Through this technology, the objects are interconnected with each other in the smart cities. However, this higher performing usage of the applications raise up with security risks and privacy issues. In this work, we present an outline of smart city with low power synthesis technique using IoT and Computer Vision and this improves the efficiency to meet the real time environment around the world.

In the future scenario, mechanisms are to be developed to transmit data with long transmission range. We also identify the risks and challenges in the IoT, yet to be solved with the application requirements. This IoT with the key feature along with intelligent systems preserve the rights of citizens for smart city.

\section{Acknowledgement}

The authors would like to gratefully acknowledge the Department of Electronics and Computer Engineering, Koneru Lakshmaiah Education Foundation, Vaddeswaram, Guntur, India for providing the evaluation and encouragement and SmartBridge Educational Services Pvt. Ltd company for providing resources for development.

\section{References}

[1] http://www.gartner.com/newsroom/id/3616417.Gartner,Inc.,Tec hnical report, 2016

[2] Khatoun, R., Zeadally, S.: 'Smart cities: concepts, architectures, research opportunities', Commun. ACM, 2016, 59, (8), pp. 46-57

[3] M. M. Rathore, A. Ahmad, A. Paul, and S. Rho, "Urban planning and building smart cities based on the internet of things using big data analytics", Comput. Netw., 2016, DOI: 10.1016/j.comnet.2015.12.023.

[4] A. Botta, W. de Donato, V. Persico, and A. Pescapé, "Integration of cloud computing and internet of things:

[5] Simplify the development of your IoT solutions with IoT architectures:IBM Watson $7^{\text {th }}$ august 2017

[6] https://www.ibm.com/developerworks/library/iot-lp201-iotarchitectures/

[7] X. Ye, and J. Huang, "A framework for Cloud-based smart home," International Conference on Computer Science and Network Technology (ICCSNT), Vol. 2, pp. 894-897, December 2011.

[8] L. Martirano, "A smart lighting control to save energy," IEEE $6^{\text {th }}$ International Conference on Intelligent Data Acquisition and Advanced Computing Systems (IDAACS), Vol. 1, pp. 132-138, September 2011.

[9] M. Castro, A. Jara, and A. Skarmeta, "Smart lighting solutions for smart cities," 27th International Conference on Advanced Information Networking and Applications Workshops, pp. 1374 1379, March 2013.

[10] Bogatinoska, D.C., Malekian, R., Trengoska, J., et al.: 'Advanced sensing and internet of things in smart cities'. 2016 39th Int. Convention Information and Communication Technology, Electronics and Microelectronics (MIPRO),2016, pp. 632-637

[11] Poon, C.C.Y., Zhang, Y.-T., Bao, S.-D.: 'A novel biometrics method to secure wireless body area sensor networks for telemedicine and m-health', IEEE Commun. Mag., 2006, 44, (4), pp. 73-81

[12] R. P. Dick and N. K. Jha, COWLS: Hardware-software cosynthesis of distributed wireless low-power embedded clientserver systems," in Proc. Int. Conf. VLSI Design, Jan. 2000.

[13] http://www.engineering-bachelors-degree.com/electroniccomponents/uncategorized/power-aware-architecturalsynthesislow-power-system-synthesis/

[14] W. He, G. Yan, and L.D. Xu, "Developing vehicular data Cloud services in the IoT environment," IEEE Trans. Ind. Inf., vol. 10, pp. $1587-1595,2014$.

[15] A. Zaslavsky, C. Perera, and D. Georgakopoulos, "Sensing as a service and big data," 2013. ArXiv Preprint arXiv:1301.0159.

[16] I.F. Akyildiz, W. Su, Y. Sankarasubramaniam, and E. Cayirci, "Wireless sensor networks: a survey," Comput. Netw, vol. 38, pp. 393-422, 2002.

[17] F. Zhao, "Sensors meet the Cloud: Planetary-scale distributed sensing and decision making," 9th IEEE International Conference on Cognitive Informatics (ICCI), pp. 998, 2010. 